\title{
The nucleotide sequence of the Tn5271 3-chlorobenzoate 3,4-dioxygenase genes (cbaAB) unites the class IA oxygenases in a single lineage
}

\author{
Cindy H. Nakatsu, ${ }^{1} \dagger$ Neil A. Straus ${ }^{2}$ and R. Campbell Wyndham ${ }^{1}$ \\ Author for correspondence: R. Campbell Wyndham. Tel: +16137882600 ext. 3651. Fax: +1613788 3539 . \\ e-mail: cwyndham@ccs.carleton.ca
}

1 Institute of Biology, Carleton University, 1125 Colonel By Drive, Ottawa, Ontario, Canada K1S 5B6

2 Department of Botany, University of Toronto, Toronto, Ontario, Canada M5S 2B3

\begin{abstract}
The nucleotide sequence of the 3-chlorobenzoate 3,4-dioxygenase genes, designated cbaAB, from the transposon Tn5271 was determined. The function of the two sequenced open reading frames was evaluated by mutagenesis and expression in vivo to show that the cbaA and cbaB genes code for dioxygenase and reductase proteins, respectively. Comparison of the deduced amino acid sequences of the cbaAB genes with sequences for other oxygenases revealed a clearly defined lineage among the class IA oxygenases that shows several unique features. This lineage includes phthalate 4,5-dioxygenase (pht23), and based on the available $\mathbf{N H}_{3}$-terminal sequence of component $\mathbf{A}$, also includes 4-sulphobenzoate 3,4-dioxygenase. Vanillate demethylase, encoded by the vanAB genes and formally a monooxygenase enzyme catalysing an oxidative demethylation, is also included in this lineage. The terminal chlorobenzoate dioxygenase (CbaA) component is characterized by a conserved Rieske-type [2Fe-2S $]_{R}$ ligand centre. The reductase component (CbaB) contains a plant-type ferredoxin [2Fe-2S $]_{\mathrm{Fd}}$ FMN-isoalloxazine and NAD-ribose-binding domains and the orientation of these domains is conserved in all known class IA reductases. These results support the hypothesis that alternative fusions of the electron transfer modules of the reductases arose early in the divergence of oxygenase systems. The over-riding evolutionary constraint acting on the divergence of the class IA oxygenases would appear to be the requirement for a carboxyl group para to the site of oxygen insertion into the aromatic ring.
\end{abstract}

Keywords: transposon, chlorobenzoate, dioxygenase, plasmid

\section{INTRODUCTION}

Complex mixtures of organic compounds found in contaminated soils and surface waters are a powerful selective force for the evolution of bacterial catabolic pathways. Many isolates have been recovered from these environments on the basis of their ability to utilize selected contaminants as their sole source of carbon and energy (Sayler et al., 1990; van der Meer et al., 1992). While the aromatic catabolic determinants of some genera

† Present address: Center for Microbial Ecology, R.O.M.E. Project, Michigan State University, East Lansing, MI 48824-1325, USA.

The GenBank accession number for the sequence reported in this paper is U00692. have been thoroughly studied, particularly the fluorescent pseudomonads and Acinetobacter (Gibson \& Subramanian, 1984; Frantz \& Chakrabarty, 1986; Reineke \& Knackmuss, 1988; Harayama et al., 1992), other genera are not well characterized. As new catabolic gene sequences from diverse contaminant-degrading species are determined, new insights into evolutionary lineages and origins of these elements are revealed (Neidle et al., 1991).

The metabolism of chlorobenzoates illustrates the diversity of pathways for contaminant degradation that is likely to be encountered in natural communities or industrial consortia. There are now several known pathways for the metabolism of chlorobenzoates among the aerobic proteobacteria. The chlorobenzoate 1,2dioxygenase pathway, wherein oxygen is introduced at the carboxyl and adjacent positions of the chlorobenzoate 
ring, forming chlorocatechols, has been thoroughly studied (Reineke \& Knackmuss, 1988; Hickey \& Focht, 1990; Harayama et al., 1992). This pathway is initiated either by benzoate(toluate) 1,2-dioxygenases with broad substrate specificity (Fetzner et al., 1992), or by ortbohalobenzoate 1,2-dioxygenases that are specific for ortbosubstituted halobenzoates (Romanov \& Hausinger, 1994). An operon representative of the former, $x y l X Y Z$ from the Pseudomonas putida plasmid pWW0, has been sequenced and the deduced amino acid sequences have been compared to the narrow-substrate-range benzoate 1,2-dioxygenase of Acinetobacter calcoaceticus (ben $A B C$ ) (Harayama et al., 1991; Neidle et al., 1991). Sequences of chlorocatechol ortho-ring-fission genes ( $\operatorname{clc} A B D)$ from $P$. putida (pAC27), required for complete metabolism of chlorobenzoates by this pathway, have also been determined. They reveal similarities to the corresponding catechol ortho-ring-fission genes found in Pseudomonas and Acinetobacter, and to other chloroaromatic ortbo-ringfission genes (Frantz \& Chakrabarty, 1987; Neidle et al., 1988; van der Meer et al., 1991, 1992; Harayama et al., 1992). Another known pathway involves the removal of a chlorine substituent by hydrolytic dehalogenation. This pathway functions in Pseudomonas sp. CBS-3 growing on 4-chlorobenzoate (Savard et al., 1986; Scholten et al., 1991). An early report on the metabolism of 3-chlorobenzoate in Pseudomonas attributed a similar transformation, to form 3-hydroxybenzoate, to a monoxygenase (Johnston et al., 1972). Very little is known concerning the biochemistry and genetics of the latter pathway. Recently we described a new pathway in Alcaligenes sp. strain BR60 that expresses a 3-chlorobenzoate 3,4-dioxygenase activity (Nakatsu \& Wyndham, 1993). This dioxygenase introduces oxygen at the carbons of benzoate distal to the carboxyl group, yielding protocatechuate and chloroprotocatechuate metabolites. The genes for this pathway are carried on a $3.7 \mathrm{~kb}$ NotI-EcoRI fragment within the catabolic transposon $\operatorname{Tn} 5271$, originally found on the $85 \mathrm{~kb}$ conjugative plasmid pBRC60 (Wyndham et al., 1988, 1994; Nakatsu et al., 1991; Nakatsu \& Wyndham, 1993).

Oxygenases that are involved in the initial attack on aromatic substrates have been grouped according to the nature of the oxidation they carry out, and according to the properties of their electron transport proteins (Harayama et al., 1992; Mason \& Cammack, 1992). The aromatic-ring-hydroxylating dioxygenases have been classified into two-component systems and threecomponent systems, based on the number of proteins involved in electron transport and hydroxylation (Fetzner et al., 1992). These broad classes have been further subdivided according to the detailed arrangements and cofactor requirements of their reductase and oxygenase components (Batie et al., 1991; reviewed by Mason \& Cammack, 1992). Briefly, these classes and their distinguishing features are: class I, two-component oxygenases in which a flavin and iron-sulphur cluster are combined in the same reductase (class IA: FMN cofactor; class IB: FAD cofactor); class II, three-component dioxygenases in which the flavin (reductases) and iron- sulphur proteins (ferredoxins) are separate (class IIA: chloroplast-type ferredoxins; class IIB: Rieske-type ferredoxins); class III, three-component dioxygenase in which the reductase and ferredoxin are separate, but the reductase contains both a flavin and an iron-sulphur cluster. All of these classes have terminal oxygenase components with two common cofactors, a Rieske-type $[2 \mathrm{Fe}-2 \mathrm{~S}]_{\mathrm{R}}$ iron-sulphur centre and one mononuclear non-haem iron.

In order to place the 3-chlorobenzoate 3,4-dioxygenase of Tn5271 into the context of the evolution of bacterial oxygenases, we determined the activity expressed by the genes designated $c b a A B$ from Alcaligenes sp. BR60, and their nucleotide sequence. From this sequence we derived putative amino acid sequences and compared these with known sequences for other oxygenases. This comparison revealed a clearly defined lineage among the biochemically defined class IA oxygenases that shows several unique features.

\section{METHODS}

Strains, plasmids and growth conditions. The growth and maintenance of Alcaligenes sp. strain BR60 (pBRC60), strain BR6024 (chloramphenicol resistant, tryptophan auxotroph) and Escherichia coli strains have been described previously (Wyndham et al., 1988; Wyndham \& Straus, 1988; Nakatsu et al., 1991; Nakatsu \& Wyndham, 1993). E. coli strain DH5 $\alpha$ was used as the host for the broad-host-range pBW13 constructs pBRCN5, pBRCN7, pBRCN11, pBRCN12, pBRCN15 and pBRCN16, containing the dioxygenase coding region of $\operatorname{Tn} 5271$ and deletion and point mutations of this region (Nakatsu \& Wyndham, 1993; this study). E. coli strain JM109 was the host for the pUC18 plasmid construct $\mathrm{pBRH} 2$, containing the cloned HindIII restriction fragment $\mathrm{H} 2$ of $\mathrm{Tn} 5271$ (Wyndham et al., 1988). E. coli strain HB101 was used as the host for singlestranded DNA synthesis from clones in M13mp18 and M13mp19 (Yanisch-Perron et al., 1985).

DNA cloning, sequencing and mutation. DNA extraction and cloning was performed according to previously described methods (Nakatsu et al., 1991). The HindIII fragment $\mathrm{H} 2$ of Tn5271, originally cloned into the HindIII site of pUC18 to give pBRH2 (Wyndham et al., 1988), was the source of the DNA sequenced in this study. Plasmid pBRH2 was digested with EcoRI and the fragments E11 $(2.9 \mathrm{~kb}), \mathrm{E} 13(1.6 \mathrm{~kb})$ and E14 $(1.5 \mathrm{~kb})$ were ligated into the replicative forms of phage M13mp18 and M13mp19 in both orientations. These constructs were transformed into E. coli $\mathrm{HB} 101$ to produce single-stranded DNA for sequencing in both directions using the commercial dideoxy chain-termination Sequenase kit (United States Biochemical). Oligonucleotides for sequencing were prepared as described by Nakatsu et al. (1991).

The $c b a$ gene region was mutagenized to determine the requirements for chlorobenzoate dioxygenase activity by creating a deletion and two site mutations, followed by the evaluation of expression in vivo. Unique Scal restriction sites within $c b a A$ were used to create a deletion of the internal fragment of pBRCN5 in vitro, by restriction digestion and ligation, followed by transformation into E. coli $\mathrm{DH} 5 \alpha$ and triparental mating into Alcaligenes sp. BR6024 (Nakatsu \& Wyndham, 1993). The only known unique site within the second ORF ( $c b a B$ ) was for EcoRV, but this site also existed within the vector $\mathrm{pBW} 13$. Therefore, the PstI-HindIII frag- 
ment of pBRH2 was cloned into pUC18, then the EcoRV site was mutated by restriction digestion and treatment with exonuclease III for $1 \mathrm{~s}$ according to the manufacturer's directions (Boehringer Mannheim). The site was then filled, religated and the plasmid was transformed into $E$. coli $\mathrm{DH} 5 \alpha$ as described (Sambrook et al., 1989). The modified fragment was then excised from pUC18, cloned into the broad-host-range vector $\mathrm{pBW} 13$ and conjugated into Alcaligenes sp. strain BR6024 as described previously (Nakatsu \& Wyndham, 1993). A third mutation was created $3^{\prime}$ to the $c b a B$ ORF by digesting pBRCN5 with $B s t$ EII and treating with exonuclease III as described above. The presence of the specified modifications to all plasmids was confirmed by plasmid isolation and restriction mapping.

Sequence analyses. The National Center for Biotechnology Information (NCBI) BLAST and BLASTX programs were used to search for similarities to the $c b a A B$ nucleotide sequences and deduced amino acid sequences (Altschul $e t$ al., 1990). The nonredundant components of peptide sequence databases, including SwissProt, PIR, GenPept (translations from GenBank) and GPUpdate (cumulative daily updates to the major release), were searched. The GCG sequence analysis programs of the University of Wisconsin (Devereux et al., 1984), provided through the Molecular Biology Database System of the National Research Council of Canada, were used for all analyses. Amino acid sequence alignments were carried out using the GAP, BESTFIT and PILEUP programs of the GCG7 package. For these alignments a gap weight of $3 \cdot 0$ and a gap length weight of $0 \cdot 1$ were used. Complete sequences were used for alignments within the class IA oxygenase group containing $\mathrm{Cba}$, Van and Pht protein sequences. Complete sequences and partial sequences were used for alignments between amino acid sequences of class IA and the other classes of oxygenases as defined by Batic et al. (1991). The sequences of the 200 amino acids spanning the Rieske-type $[2 \mathrm{Fe}-2 \mathrm{~S}]_{\mathrm{R}}$ domains of the terminal oxygenase components were aligned to show similarities across dioxygenase classes. Similarly, 200 amino acids containing the contiguous FMN- (FAD-) isoalloxazine binding and NAD-ribose-binding domains of the reductase components, and 110 amino acids spanning the plant ferredoxin-like $[2 \mathrm{Fe}-2 \mathrm{~S}]_{\mathrm{Fd}}$ domains of these same components, were aligned individually. The pILEup program uses a progressive alignment method the order of which is based on pairwise similarity scores for each pair of sequences. The pairwise similarity scores were used to cluster the sequences by similarity. Relative evolutionary distances were presented graphically as dendrograms using these pairwise similarity scores.

\section{RESULTS}

\section{Localization and nucleotide sequence of the cbaAB genes}

A restriction digest map of $\operatorname{Tn} 5271$, showing the flanking IS 1071 elements and the non-repeated region carrying the $c b a A B$ (3-chlorobenzoate 3,4-dioxygenase) genes is presented in Fig. 1. This figure illustrates the subclones constructed to determine the function of the $c b a A B$ genes.

The nucleotide sequence from position 4600 to 6938 is presented in Fig. 2. The nucleotide numbering system begins with nucleotide 1 as the first $G$ in the left inverted repeat of the IS1071 L element of Tn5271 (Fig. 1; GenBank accession number M65135; Nakatsu et al., 1991). Previous studies (Nakatsu \& Wyndham, 1993) demonstrated that unique protein products were produced only in the left to right (PstI to HindIII) orientation, the upper strand in this study, therefore only the deduced amino acid sequences of the ORFs of the upper strand are included in Fig. 2. The ORFs were designated $c b a A$ and $c b a B$.

Conjugation of the $\mathrm{pBRCN} 5$ construct containing these ORFs plus flanking DNA into Alcaligenes sp. BR6024 restored the ability of this mutant to grow on 3chlorobenzoate. Elimination of the ScaI fragment within the $c b a A$ ORF to give the $\mathrm{pBRCN} 7$ plasmid resulted in a failure to restore the degradative phenotype.

The clone pBRCN15 was created by eliminating the EcoRV restriction enzyme site within $c b a B$ of $\mathrm{pBRCN} 5$ by exonuclease digestion. The introduction of this plasmid into Alcaligenes sp. strain BR6024 did not initially produce 3 -chlorobenzoate-degrading transconjugants. After prolonged incubation (3 weeks), colonies did appear on the 3chlorobenzoate selection plates. When these putative transconjugants were patched to fresh 3-chlorobenzoate selection plates, growth of $10 \%(5 / 50)$ of the patched colonies occurred. If the tetracycline resistance marker of pBRCN15 was used for selection of transconjugants on succinate plates, followed by patching of transconjugants onto 3-chlorobenzoate, no growth occurred even after prolonged incubation. These observations suggest $\mathrm{CbaB}$ is required for 3-chlorobenzoate 3,4-dioxygenase activity, and that we observed mutational recovery of this activity due to repair of the deletion around the EcoRV site in vivo.

The clone pBRCN16 was created by eliminating the $B s t$ EII restriction enzyme site that lies approximately $0.3 \mathrm{~kb}$ beyond the stop codon of $c b a B$. The clones pBRCN11 and pBRCN12 were created by nested deletions in the same region of DNA, $3^{\prime}$ to the $c b a B$ gene (Nakatsu $\&$ Wyndham, 1993). The introduction of these plasmids into Alcaligenes sp. strain BR6024 did not produce 3chlorobenzoate-degrading transconjugants. However, the pBRCN16 and pBRCN11 transconjugants metabolized 3-chlorobenzoate to dihydrodiol intermediates that were unstable (Nakatsu \& Wyndham, 1993). The major product accumulating in the medium of these transconjugants was a chlorohydroxybenzoate, presumed to be the product of spontaneous dehydration of a chlorodihydrodiol intermediate. The $\mathrm{pBRCN} 12$ transconjugants failed to metabolize 3-chlorobenzoate, indicating that the nested deletion had eliminated an essential sequence required for dioxygenase expression. Restriction mapping revealed that this deletion extended into the $c b a B$ gene.

\section{Sequence characterization}

The first ORF, $c b a A$, has potential initiation codons at nucleotides 4631, 4685 and 4697 of Tn5271 (Fig. 2). The potential ribosome-binding site preceding nucleotide 4631 is AGGAG, very close to the ribosomal-binding site consensus sequence for $E$. coli, therefore this was chosen as a tentative initiation codon. The $c b a A$ gene potentially codes for a protein of 432 amino acids with a deduced molecular mass of $48932 \mathrm{Da}$. The second ORF, $c b a B$, has 
(a)

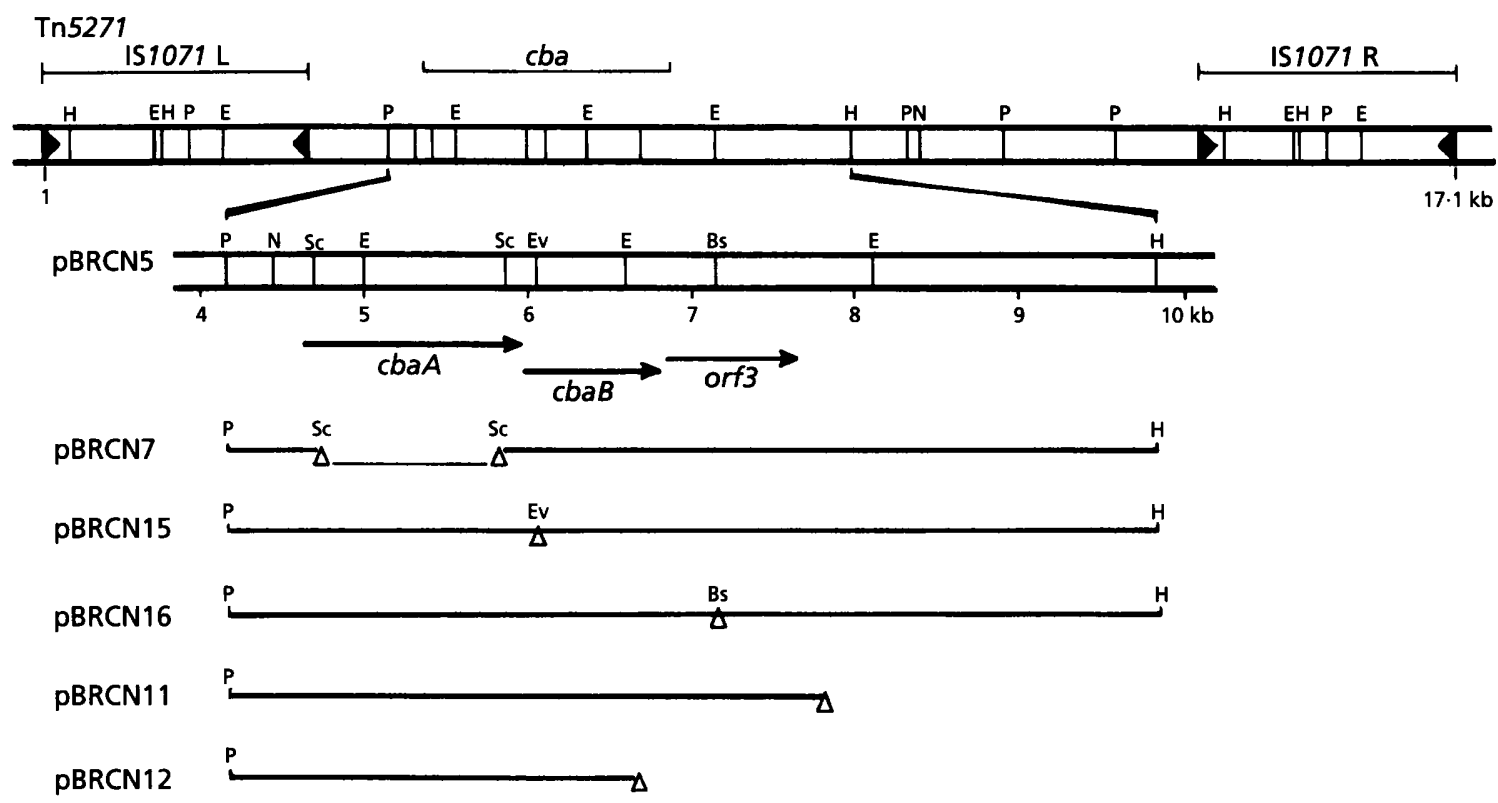

Fig. 1. Restriction enzyme digest map of Tn5271 showing the location of the $c b a A$ and $c b a B$ genes. (a) Map of the entire Tn5271 element. Restriction enzymes used were: Bs, BstEll; E, EcoRI; Ev, EcoRV; H, HindllI; N, Notl; P, Pstl; Sc, Scal. (b) The region of Tn5271 cloned into the IncP $\beta$ broad-host-range vector pBW13 to form pBRCN5. The $c b a A$ and $c b a B$ genes are designated by arrows (encoded on the upper strand as drawn, with the $5^{\prime}$ end to the left). Downstream of $c b a B$ there is another ORF (ORF3) not fully characterized. Distances are marked in kb relative to the numbering system for Tn5271, with nucleotide 1 being the first $\mathrm{G}$ in the left inverted repeat of $151071 \mathrm{~L}$ (Nakatsu et al., 1991). The mutations of pBRCN5 used to determine functions of the ORFs are shown below. The construction of pBRCN7, pBRCN11 and pBRCN12 was previously described (Nakatsu \& Wyndham, 1993). The pBRCN15 and 16 mutations were created by deletion of nucleotides at restriction enzyme sites for EcoRV and BstEIl, respectively.

an initiation codon at nucleotide 5983 with a potential ribosome-binding site with the sequence GGAGG. The $c b a B$ gene potentially codes for a 288 amino acid protein with a deduced molecular mass of $31704 \mathrm{Da}$.

The $\mathrm{G}+\mathrm{C}$ composition of $c b a A$ is $58.7 \%$ and of $c b a B$ is $59.2 \%$, in the expected range for the Alcaligenes sp. BR60 family Comamonadaceae $(57-70 \% \mathrm{G}+\mathrm{C}$ ) (Willems et al., 1991). This $\mathrm{G}+\mathrm{C}$ composition is similar to the $58.8 \%$ $\mathrm{G}+\mathrm{C}$ content for the IS1071 transposase sequence flanking cba $A B$ within Tn5271 (Nakatsu et al., 1991). There is very little information on codon usage by species within the Comamonadaceae, however we can comment on the differences in codon usage when compared to codons used by fluorescent Pseudomonas species (West \& Iglewski, 1988). For example, arginine AGA and AGG are used nine times in the $c b a A B$ genes, which is unlike codon usage in the fluorescent pseudomonads. Harayama et al. (1991) found that there was an exceptional use of these same codons in the $x y / X$ gene of $P$. putida, prompting them to speculate on alternative origins for this gene. Codons considered rare in Pseudomonas aeruginosa, but used frequently in $c b a A B$ are: arginine-CGA, alanine-GCA, isoleucine-ATT, leucine-TTG, glycine-GGG and GGA, and valine-GTT. In cba $A$ alanine-GCT and arginineCGT and AGG, and in $c b a B$ asparagine-AAT, cysteineTGT, isoleucine-ATA and serine-AGT, are all rare codons for Pseudomonas that are used in the cba $A B$ genes.
These codon usage patterns suggest the $c b a A B$ genes do not derive from fluorescent Pseudomonas species.

\section{Alignments of oxygenase amino acid sequences}

The BLASTX search of peptide sequence databases for similarities to the $c b a A$ gene product listed the products of $p b t 3$ and $\operatorname{van} A$ as the closest matching full sequences (Fig. 3). The pht12345 genes were described by Nomura et al. (1992). They are found on a $7 \mathrm{~kb}$ EcoRI fragment of the PHT plasmid of $P$. putida strain NMH102-2 and their expression allows this strain to grow with phthalate as sole source of carbon. The chromosomal van $A B$ genes encode vanillate demethylase activity in a Pseudomonas species (Brunel \& Davison, 1988). The latter activity has been described as a monooxygenase-catalysed oxidative demethylation. In addition to the above matches, similarity was also detected to a partial amino acid sequence of the $\mathrm{NH}_{3}$-terminal 35 residues sequenced from the purified component A of the 4-sulphobenzoate 3,4-dioxygenase of Comamonas testosteroni strain T2 (Locher et al., 1991). The $\mathrm{NH}_{3}$-terminal sequence of the 4-sulphobenzoate 3,4dioxygenase showed $48 \%$ identity to $\mathrm{CbaA}$ over the available 35 amino acid sequence. This was a much greater similarity than for the $\mathrm{CbaA} / \mathrm{Pht} 3$ comparison in the same region of the sequence $(37 \%)$. This suggests that when the complete 4-sulphobenzoate 3,4-dioxygenase sequence 
$C D a A B$

4620

4631

AGGAGCAAGCT ATG GCA TTG ACC AAG GAC AAC GAA GAT TG GTG CGA GTG GGC CGT [CDaA] het Ala Leu Thr Lys Asp Asn Glu Asp Leu Val Arg Val Gly Arg

GGA ACC CCG ATG GGC GGC CTC ATG CGC GAG TAC TGG ATC CCC GCA TTG AAA TCG Gly Thr Pro Met Gly Gly Leu Het Arg Glu Tyr Trp Ile Pro Ala Leu Lys Ser 4730

ACC GAG CTC GAG GCT GGC GGA AGT CCT GTG AGG CTG CTG CTC TTG GGC GAA AAG Thr Glu Leu Glu Ala Gly Gly Ser Pro Val Arg Leu Leu Leu Leu Gly Glu Lys 4784

CTC GIC GCC TIC AGG GAG CCG TCG GGT GCG GTC GGC GTG ATG GAC AGC CGA TGC Leu Val Ala Phe Arg Glu Pro Ser gly Ala Val Gly Val Met Asp Ser Arg Cys 4838

CCC CAC CGT GGC GT TCC CTG TT ATG GGG CGC GTT GAA GAG GGC GGG CTG CGC Pro His Arg Gly Val Ser Leu Phe Met Gly Arg Val Glu Glu Gly Gly Leu Arg 4892

TSCC GTC TAC CAC GGT TGG AMA TTC AGC GCT GAG GGC AAA TGC GTT GAC ATG CCC Cys Val Tyr His Gly Trp Lys Phe Ser Ala Glu Gly Lys Cys Val Asp Met Pro

AGT GIC CGT CCT GAA GAC GAA TTC AAA AAC AGC GTG CGC GTG GCG CGA TAC CCG Ser Val Arg Pro Glu Asp Glu Phe Lys Asn Ser val Arg Val Ala Arg Tyr Pro 5000

STOG AMA GAA ATG GCG GGC GIT GTC TGG GTG TAC ATG GGT ACT CGA AAA GTT TTG Val Lys Glu Met Ala Gly Val Val Trp Val Tyr Met Gly Thr Arg Lys Val Leu

CCC GAG CTC CCG CGG CTG GAA GTG CTG AAT CT CCG GAA AAC GAA GTG GAT GTC pro glu Leu pro arg Leu glu Val Leu Asn Leu Pro glu Asn glu Val Asp Val 5108

ATT TGC CTC CAG CGA AAG TCG AAC TGG ITG CAA AAC CTG GAA GGT GAA ATT GAT Ile cys Leu Gin Arg Lys Ser Asn Trp Leu Gin Asn Leu Glu Gly Glu Ile Asp 5162

ACC TCG CAT TTC AAC TTC CTT CAC GTG GGC GGA CTC CAC GCG GAC GAA GTG CCG Thr Ser His phe Asn phe Leu His Val gly gly Leu His Ala Asp glu Val Pro 5216

GAC GAT CAC CCA TTG AAA TAC ACC GCC CAA GTC GCT CCG CAG TAC CTG GTC AAG Asp Asp His pro Leu Lys Tyr Thr Ala Gin Val Ala Pro Gln Tyr Leu Val Lys 5270

GAA ACG GCA TTG GGC ACC TGC TAC GCG GCT CAA GTG CCG GCG GAA GAG GAT CAC Glu Thr Ala Leu Gly Thr Cys Tyr Ala Ala Gin Val pro Ala Glu Glu Asp His 5324

ACC TAC ACG CGG TIT GCG CAT TTC CT TC CCG TC TGG GCG CTC ATT CCG CAA Thr Tyr Thr Arg Phe Ala His phe Leu Phe Pro Phe Trp Ala Leu Ile Pro Gin 5378

GCC GAT ATT GCG CAG AAC ATT TTG GCC AGA GCC TGG GTG CCC ATG GAC GAT GAG Ala Asp Ile Ala gin Asn Ile Leu Ala Arg Ala Trp Val pro Met Asp Asp glu 5432

CAC ACC ATG ATG TTC ITC TIC CGT TGG ACG GGA TCC AAG GCC AAA CGC CTC GAT His Thr Met Met Phe Phe Phe Arg Trp Thr Gly Ser Lys Ala Lys Arg Leu Asp 5486

ACG CCA TTG AAG AGC GGT TCG CCC ATG CCC GGG GTC ACG CTG ACG GAC ATG AAG Thr Pro Leu Lys Ser Gly Ser Pro Met Pro Gly val Thr Leu Thr Asp Met Lys 5540

TAC AAG GAA AAC ACG ACG GAT TGG TAC GGC CGC TGG CAG CCG CTC GGC GAT GAG Tyr Lys Glu Asn Thr Thr Asp Trp Tyr Gly Arg Trp Gin pro Leu Gly Asp glu 5594

AGC AAC GAT TGG CTC ATC GAT CGA GAT TTG CAA AAA GTA GGC CGG GTG TTC TCC Ser Asn Asp Trp Leu lle Asp Arg Asp Leu Gin Lys Val Gly Arg Val phe Ser 5648

GGT ATT TAC GGA ATC CAC GCA CAG GAT CAG GCC ATG ACC GAC AGC ATG GGG CCG Gly Ile Tyr Gly Ile His Ala Gln Asp Gln Ala Met Thr Asp Ser Met Gly Pro 5702

ATC ATT GAC CAC GGC CTG GAG CAA TIG GCA CCG ACC GAT CTG ATG ATC GTC AGA Ile Ile Asp His Gly Leu Glu Gln Leu Ala Pro Thr Asp Leu Met Ile Val Arg
ACC CGT CGC CGG ATT CTC AAG GCG CTC CGT GCG CAC GAA GCG AAT GGC ACC CTG Thr Arg Arg Arg Ile Leu Lys Ala Leu Arg Ala His Glu Ala Asn Gly Thr Leu

5810 CCC GGT GTT GAC GAG GCG GAT CAG TAC TC ACG CCC AGG AGC GGC TAT TAC pro pro gly Val Asp Glu Ala Asp Gln Tyr phe Thr pro Arg Ser Gly Tyr Tyr

CTC ACG CCG AAA AGC GTC GAC TGG GAG ACC GCT TAC GAA CAA AGG ATC GAG GGC Leu Thr Pro Lys Ser val Asp Trp Glu Thr Ala Tyr Glu Gin Arg Jle Glu Gly 5918
CTC GTG CGG TGA
5929 CTC GTG CGG TGA

Leu Val Arg * 5983

5968 CGCGGAGTGATGCC 5983 ATG GTC GCA ATC GAT CAA CAC GAT ACC TAC AGT GTT CGG GTG [CbaB] het Val Ala lle Asp Gin His Asp Thr Tyr Ser Val Arg Val

6025

ATA TCC CGA TCG CAC CTG AGC AAG GAT ATT GTT CAG GTG GAA CTC GAG GAA TCG Ile Ser Arg Ser His Leu Ser Lys Asp Ile Val Gin val Glu Leu Glu Glu Ser 6079

AGC CAG CGA CCA TTG CCG GAC TAT GAG CCG GGA TCG CAC GTA GAC ATC TAT GTC Ser gin arg Pro Leu Pro Asp Tyr Glu Pro Gly Ser His Val Asp Ile Tyr Val 6133

CAG GAT GAC CTT GTC CGG CAA TAC TCG CTG GTC AAG GCG TCC GAC GCG CAG GCC Gin Asp Asp Leu Val Arg Gin Tyr Ser Leu Val Lys Ala Ser Asp Ala Gln Ala

T187 TAC CAG ATC GCC TTC AAG GTC AAG CGC GAT CAG GGC AGT GCG ACG GAG CTG Ser Tyr Gin Ile Ala phe Lys Val Lys Arg Asp Gin Gly Ser Ala Thr Glu Leu

A241 TGC GAG TTG CTC CGT GTC GGT GCA ACA ACC CGA ATC TCT GCG CCG AGG AC Met Cys Glu Leu Leu Arg Val Gly Ala Thr Thr Arg lle Ser Ala Pro Arg Asn

6295 Ala Phe Ala Leu Asp Pro Gln Ala Arg Glu Thr val Leu Ile cys Gly Gly Ile 6349

GGA ATA ACG CCC ATG GTT CAT ATG GCC ATG ACC CTG GTC AAA GCG AAG CGG CCA Gly Ile Thr pro met Val His Met Ala Met Thr Leu Val Lys Ala Lys Arg Pro 6403

TGG AGC ATG CAC ATT GCC TCC CGG GAT GGC GAT GAG CTC GAT TTG CTG GGG CCG Trp Ser Met His Ile Ala Ser Arg Asp Gly Asp Glu Leu Asp Leu Leu Gly Pro 6457

CTG GGA AGT TGC TCC GAG ATC AGC CGC TAC ATC TCG ICC CAG GGG GAC AGA TTG Leu Gly Ser Cys Ser Glu Ile Ser Arg Tyr Ile Ser Ser Gln Gly Asp Arg Leu 6511

CCG ATT CGG GAC CTG GCC GAG CGC GCA CCT GCG AAT GCG CAT CTG TAC TTC TGC Pro Ile Arg Asp Leu Ala Glu Arg Ala Pro Ala Asn Ala His Leu Tyr Phe Cys 6565

6565 CCT GAG GGC ATG TTG CAG GAA TTC ACG GTG AAT CT GCC CGC AGT GGG GCG Gly Pro Glu Gly Met Leu Gln Glu Phe Thr Val Asn Leu Ala Arg Ser Gly Ala

CAG TAT GTC GTG CGC GAG GGC GAG ACG ATA TTG GAT GTC CTG CGC AAT GCC GGC Gin Tyr Val Val Arg Glu Gly Glu Thr Ile Leu Asp Val Leu Arg Asn Ala Gly 6673

CAC CAT GTC ACG TCC TCC TGT CGG CAG GGG ATT TGT GGC ATG TGT GAA ACC ACT His His Val Thr Ser Ser cys Arg Gin Gly Ile Cys Gly Met Cys Glu Thr Thr 6727

ITG ATT TCC GGC GTG CCG GAC CAC AGG GAC CGC TGG CTG ACC GAT AGC GAA AAG Leu Ile Ser gly Val Pro Asp his arg Asp Arg Leu Leu Thr Asp Ser Glu Lys 6781

GCC TCG GGT CGA ACC ATG TTG ATT TGC TGT TCA CGC GCG CTC AGC CCG GAA CTC Ala Ser Gly Arg Thr Met Leu Ile Cys Cys Ser Arg Ala Leu Ser Pro Glu Leu ACC CTG GAT CTG TGA AT ATG AGA ATA GGG GTC GTG GGC CTG GGG AAG GCA Thr Leu Asp Leu * [ORF3] Met Arg Ile Gly Val Val Gly Leu Gly Lys Ala 6885 ATG CTG ATG CTG CCG ACG TIT CTC ATG GAT CGG CGG GTT CAA CTG GTC GCT phe Met Leu Met Leu Pro Thr Phe Leu Met Asp Arg Arg Val Gln Leu Val Ala

Fig. 2. Nucleotide and deduced amino acid sequence of the $c b a A$ and $c b a B$ genes. The nucleotide sequence of the upper strand of Tn 5271 between nucleotide positions 4600 and 6938 (nucleotide 1 defined as the first $G$ in the sequence of the left 151071 element of Tn5271; Nakatsu et al., 1991) is shown. Potential ribosomal-binding sites are underlined and initiation codons are in bold letters. Stop codons are indicated by an asterisk. The first 87 nucleotides of an ORF downstream of $c b a B$ that has been partly characterized are also shown.

is eventually determined, the alignment with $\mathrm{CbaA}$ will likely be very strong.

Fig. 4(a) shows an alignment of amino acids 50-108 of $\mathrm{CbaA}$ with similar regions of the terminal oxygenase components representing several other oxygenase classes. Within this region there is $58 \%$ identity between $\mathrm{CbaA}$ and $\mathrm{Pht} 3$, and $35 \%$ identity between $\mathrm{CbaA}$ and $\operatorname{VanA}$. Alignments in this region are similar to previously determined conserved regions containing the proposed cysteine and histidine ligands of Rieske-type $[2 \mathrm{Fe}-2 \mathrm{~S}]_{R}$ centres of several dioxygenases (Neidle et al., 1991 ; Mason \& Cammack, 1992). In addition to the Rieske-type $[2 \mathrm{Fe}-2 \mathrm{~S}]_{\mathrm{R}}$ cofactor, class IB terminal oxygenase components have been shown to bind mononuclear, non- haem $\mathrm{Fe}(\mathrm{II})$ (Yamaguchi \& Fujisawa, 1982). This cofactor is thought to be involved in oxygen binding (Mason \& Cammack, 1992). Histidine and tyrosine residues conserved in the class IB, IIB and III oxygenases have been proposed to coordinate this $\mathrm{Fe}(\mathrm{II})$ (Neidle et al., 1991). In the class IA alignment of Fig. 3, an aspartate and two histidine residues are conserved in a $\mathrm{D}-\mathrm{x}-\mathrm{x}-\mathrm{H}-\mathrm{x}-\mathrm{x}-\mathrm{x}-\mathrm{x}-\mathrm{H}$ motif in about the same position relative to the Rieske domain (separated by $90-100$ amino acids) as in the other oxygenases. No tyrosine residues are conserved in this region.

The relative evolutionary distances of the terminal oxygenase components from different classes were determined by aligning the $\mathrm{NH}_{3}$-terminal 250 amino acids of 
CbaA MaltkoNedL vRVGrGTPMG gLMReyWiP. ALkStEIEag GSPVRIILIG

Pht3

VanA

Cons

CbaA

Pht3

Vana

Cons

CbaA

Pht3

VanA

Cons

CbaA

VanA

Cons

CbaA

Pht3

VanA

Cons

CbaA

Pht3

VanA

Cons

CbaA

Pht3

VanA

Cons

CbaA

Pht3

VanA

Cons

CbaA

Pht3

VanA

Cons

CbaA

VanA
MLTpEeNILL CRVegdaPMG qHiRrhitPv cLLeEvsEpd GtPVRarLfG

MLTSEnNqLL tRVGpGTaMG $\times L$ fRhf $\times q P$. ALLSEE...

EkLVaFReps GaVGVMDSrC PHRGVSL fmG RvEeGGLRCV YHGW 99 EdLVFFRttd GRVGVMDeYC PHRrVSL iYG RUEESGLRC1 YHGWKDVDG ErmViyRgag oRVaaledfC PHRGapLsiG sigdGkLVCg YHGIVMDCDG $E V \underset{R}{V}$ C PHR L G $L \underline{C}$ YHG G

100 . 146

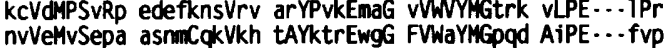
rtasMPaqRv qafpC...-i rAfPagErhG FiWwpG-da ALadpal iPh 147$$
\text { M aqRv qafpC...-i rAfPaqErhG FiWWPG }
$$

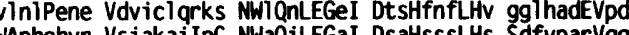
(D) EWAenPawa yggglyhIaC dyrimichlm DlthetyvHa SsigakEi..

197 D $\underline{H}$ H

.....Lk ytagvAPqyl VkeTAlGtcY AA-.. qvpAe edhtYtRfah DEApvstrve gstrlitgRfm VerTsyGFrY AALRrpigna atseyroil 239 AA

Fl fPfWALIP qadiAgnila rawVPmDDeH TmmfFfritg skakr-10TP FvaPatALIP pnnlyn--vA ninVPiDDtH TafyfmaWgn pdntpeteTw

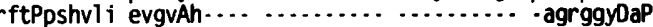
288 $P$

IKsgspmpGV tLtDinkyken ttdwyGrwqP LgdEsNdwLi DRdl qKvGrv rKflgqqvGi DLdD..............sYRP LRndgNrffq DReamknGn. adckassivV Dfitpetdts iwyfugmaRs fRpEdke-Lt aRiregqGti 338

FsGIYGiha0 DOAMtdsMGP II FtGIkGfpno DiAtwvtMGP IaDRsdErLg asDLavVefR RvmLdALaAf FaedlemieQ qQ -..rnila wpeRpLlkLn - iDaggVasR Ri IerlvsAe 388 388

eAngtlppGv deadqyftpR sgyy - -1tpK svDWetayeq riegLvr qAgE-..sai gtgekaipsR icsfqaivsk diDWrdyqar mwaLddani rAaEaqliGr qa

vaepdyevht

Fig. 3. Alignment of $\mathrm{CbaA}$, Pht3 (Nomura et al., 1992) and VanA (Brunel \& Davison, 1988) amino acid sequences. The three complete sequences are shown, along with the first 35 amino acids of component $A$ of the 4-sulphobenzoate 3,4-dioxygenase (SbaA; Locher et al., 1991). Amino acids conserved in two or more sequences are shown in upper case, and the identity consensus sequence is shown on the lower line (Cons). -, Gap introduced into the alignment. Amino acid numbers refer to the $\mathrm{CbaA}$ sequence only. Cysteine and histidine residues conserved in the Rieske [2Fe-2S] $]_{\mathrm{R}}$ domain are underlined beginning at $\mathrm{CbaA}$ amino acid 69 (see also Fig. 4). Aspartate and histidine residues conserved at the proposed mono-nuclear, non-haem $\mathrm{Fe}(\mathrm{II})$-binding domain are underlined beginning at Cba amino acid 177. Arginine residues potentially involved in the active site binding of the carboxyl group of the different substrates are underlined at $\mathrm{Cba}$ amino acids 329, 377 and 378.

each of the nine oxygenase components listed in Fig. 4(a) and plotting a dendrogram using pairwise similarity scores. This eliminates the non-conserved carboxyterminal portion of each oxygenase from consideration, weighting only the $[2 \mathrm{Fe}-2 \mathrm{~S}]_{\mathbf{R}^{-}}$and $\mathrm{Fe}(\mathrm{II})$-binding domains. The resulting dendrogram is presented in Fig. 4(b).

The second component, $\mathrm{CbaB}$, had the highest similarity scores to the reductase components of phthalate 4,5dioxygenase (Nomura et al., 1992) and vanillate demethylase (Brunel \& Davison, 1988). There are three regions of conserved sequence in these components. The first two (a)

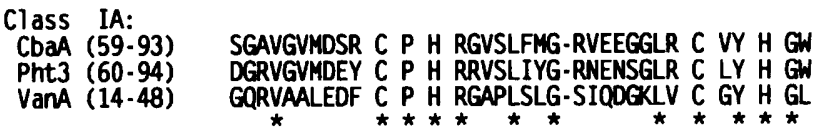

Class IB:

BenA $(85-120)$

XylX (80-115)

Class IIB:

BphA $(90-125)$

TodC1(86-121)

BnzA (86-121)

Class III:

NdoB $(71-106)$

NGELNAMINA C S H RGAQLLGHKRGKTTYYT C PF H GW DGELNAFVNA C S H RGATLCRFRSGKKATHT C SF H GW

DKSIKVFLMO C R H RGYRICRSDAGNAKAFT C SY H GW DASIAVFLNO C R H RGMRICRADAGNAKAFT C SY H GW DASIAVFLNQ C $S$ H RGMRICRADAGNAKAFT C SY H GW

\section{DGSIRAFLNV $\underset{\star}{C} R_{\star}^{H}$ RGKTLVSVEAGNAKGFV $\underset{\star}{C} S Y \underset{\star}{\text { S }}$ GW}

(b)

$[2 \mathrm{Fe}-2 \mathrm{~S}]_{R}-$ Peptide

Class

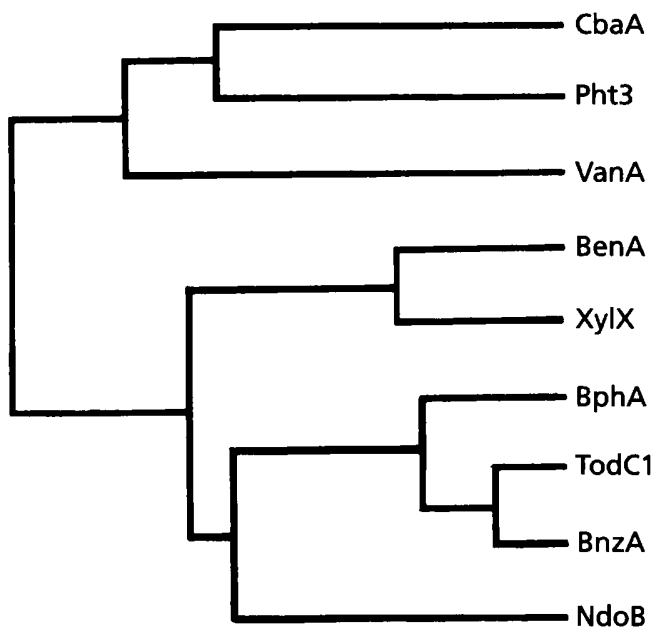

IA

IA

IA

IB

IB

IIB

IIB

IIB

III

Fig. 4. (a) Alignment of the conserved Rieske $[2 \mathrm{Fe}-2 \mathrm{~S}]_{R}$ centres of the oxygenase components of cis-diol-forming oxygenases. The class designation for oxygenases is based on the system of Batie et al. (1991). The two cysteine and two histidine residues are known to be the ligands of the $[2 \mathrm{Fe}-2 \mathrm{~S}]_{R}$ centre. *, Amino acids above this position are conserved in all of the aligned sequences; -, gap introduced into the alignment. Spaces flanking conserved residues are used for emphasis only. $\mathrm{CbaA}$, chlorobenzoate dioxygenase (this study); Pht3, phthalate 4,5dioxygenase (Nomura et al., 1992); VanA, vanillate demethylase (Brunel \& Davison, 1988); BphA, biphenyl dioxygenase (Erickson \& Mondello, 1992); TodC1, toluene dioxygenase (Zylstra \& Gibson, 1989); NdoB, naphthalene dioxygenase (Kurkela et al., 1988); BenA, benzoate dioxygenase (Neidle et al., 1991); XylX, toluate dioxygenase (Harayama et al., 1991); BnzA, benzene dioxygenase (Irie et al., 1987). (b) Relative evolutionary distances of terminal oxygenase components determined using the $\mathrm{NH}_{3}$-terminal 250 amino acids of all sequences and drawn as a dendrogram based on pairwise similarity scores.

are short regions containing consensus amino acid sequences for the binding of flavin and nicotinamide cofactors (Neidle et al., 1991). These regions of similarity are shown in Fig. 5(a, b), aligned with sequences of class IB reductase components showing similar cofactor binding regions. Also shown are the corresponding sequences for the xylene monooxygenase reductase (XylA). A third region of conserved sequence includes four cysteine 
(a)

FMN(FAD)-Isoalloxazine ring binding domain.

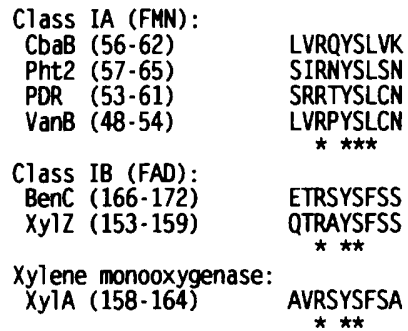

(b)

NAD-ribose binding domain.

\section{Class IA:}

CbaB (112-134)

Pht2 (114-136)

PDR (111-139)

$\operatorname{VanB}(106-126)$

ARETVLICGGIGITPMVHMAMTLVKAKRP AROFIFVAGGIGITPILSMMRHLKASTDL AKSF ILVAGGIGITPMLSMARQLRAEGLR SSRSLLFAGGIGITPILAMAQVLAARGDT

$2^{\circ}$ Structure: [

Class IB:

BenC (219-242) KRPVLMLAGGTGIAPFLSMLQVLEOKGSE

XylZ (206-229) KRPLLLLAGGTGLAPFTAMLEKIAEQGGE

Xylene monooxygenase: Xy1A $(216-238)$

NATMVCVAGGTGLAPIKCVLQSMTQAQRE

(c)

$[2 \mathrm{Fe}-2 \mathrm{~S}]_{\mathrm{Fd}}$-binding domain.

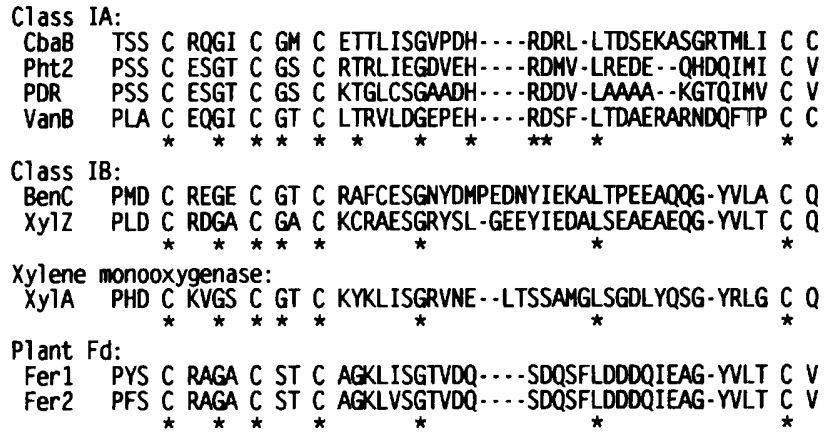

Fig. 5. Conserved regions in the reductase component of dioxygenases. *, Amino acids above this position are conserved in all of the aligned sequences above; -, gap introduced into the alignment. Spaces flanking conserved residues are used for emphasis only. $\mathrm{CbaB}$, chlorobenzoate dioxygenase reductase (this study); Pht2, phthalate 4,5-dioxygenase reductase (Nomura et al., 1992); PDR, phthalate dioxygenase reductase (Correll et al., 1992) (note that the sequences reported for the PDR protein were determined by peptide sequencing or were inferred from electron density maps); VanB, vanillate demethylase reductase (Brunel \& Davison, 1988); BenC, benzoate dioxygenase reductase (Neidle et al., 1991); XylZ, toluate dioxygenase reductase (Harayama et al., 1991); XylA, xylene monooxygenase reductase (Suzuki et al., 1991); Fer1. ferredoxin, Fischerella sp. (Hase et al., 1978; GenBank accession number A00252); Fer2, ferredoxin, Anabaena variabilis (Chan et al., 1983). (a) The FMN(FAD)-isoalloxazine ring-binding region. The numbers in brackets indicate the amino acid number. (b) The NAD-ribose-binding region. The numbers in brackets indicate the amino acid number. (c) Conserved cysteine residues involved in binding the $[2 \mathrm{Fe}-2 \mathrm{~S}]_{\mathrm{Fd}}$ centre characteristic of planttype ferredoxins. Amino acid residues used in these alignments: CbaB (234-276); Pht2 (272-312); PDR (269-309); VanB (260-302); BenC (48-94); XylZ (37-82); XylA (49-88); Fer1 (38-80); Fer2 (38-80). residues characteristic of the chloroplast-type ferredoxin $[2 \mathrm{Fe}-2 \mathrm{~S}]_{\mathrm{Fd}^{-}}$-binding domain (Otaka \& Ooi, 1989). Fig. 5 (c) shows an alignment of the class IA and IB sequences in this region with the xylene monooxygenase reductase and plant ferredoxin sequences.

In order to evaluate relative evolutionary distances of the reductase components, the protein sequences were split into two separate parts. This was necessary because the electron transfer components of different reductases have been found to be organized in modular structures that are fused in different ways to form the primary structure (Correll $e t$ al., 1992). The first part examined included the FMN/FAD-isoalloxazine- and NAD-ribose-binding domains together in sequences averaging 200 amino acids in length. The second part contained the $[2 \mathrm{Fe}-2 \mathrm{~S}]_{\mathrm{Fd}^{-}}$-binding domains of the reductases and two plant ferredoxins and averaged 110 amino acids in length. Fig. 6(a) presents a scheme of alignments between two class IA reductases (VanB and $\mathrm{CbaB}$ ) and a class $\mathrm{IB}$ reductase $(\mathrm{BenC})$ to illustrate the alternative arrangements of the conserved regions containing FMN/FAD-isoalloxazine-, NAD-ribose-, and ferredoxin-like $\left([2 \mathrm{Fe}-2 \mathrm{~S}]_{\mathrm{Fd}}\right)$-binding domains (Neidle et al., 1991 ; Correll et al., 1992). Fig. 6(b) illustrates the relative evolutionary distances of these and other class IA and IB dioxygenase reductases as dendrograms based on pairwise similarity scores for the FMN/FAD-isoalloxazine- and NAD-ribose domain (left), and ferredoxin-like $\left([2 \mathrm{Fe}-2 \mathrm{~S}]_{\mathrm{Fd}}\right)$ domain (right). Xylene monooxygenase reductase (XylA) was included in these alignments as an outlier as it has previously been shown to contain the three conserved domains shown in Fig. 5, in the same orientation as the class IB reductases (Neidle $e t$ al., 1991).

Class IIB reductase components from the biphenyl and toluene catabolic pathways (BphG and TodA, respectively) and their associated ferredoxin sequences $(\mathrm{BphF}$ and TodB) (Furukawa et al., 1989; Zylstra \& Gibson, 1989; Erickson \& Mondello, 1992) showed very little similarity to the reductase components of the class IA oxygenases (data not shown). No similarity was found in the alignments in the region expected for the FMN/FADisoalloxazine consensus. Some similarity was detected between the potential NAD-ribose-binding domain shown in Fig. 5(b), and the second dinucleotide-binding $\beta \alpha \beta$-fold described for the TodA sequence (Neidle et al., 1991 ; Mason \& Cammack, 1992). The ferredoxin domains were not similar. This was expected because the class IIB ferredoxins have spectroscopic properties and consensus sequences characteristic of Rieske-type $[2 \mathrm{Fe}-2 \mathrm{~S}]_{\mathrm{R}}$ proteins as opposed to chloroplast-type ferredoxin $[2 \mathrm{Fe}-2 \mathrm{~S}]_{\mathrm{Fd}}$ proteins (Morrice et al., 1988; Mason \& Cammack, 1992).

Another ORF, tentatively designated $c b a C$, has been identified from DNA sequence data extending beyond the $c b a B$ gene (data not shown). Mutation of this region of the cloned chlorobenzoate dioxygenase genes of pBRCN5 resulted in metabolism of 3-chlorobenzoate to dihydrodiol intermediates (results presented above). Expression studies using the thermally regulated $\mathrm{T} 7$ polymerasepromoter of pGEM3Zf indicated that the region down- 
(a)
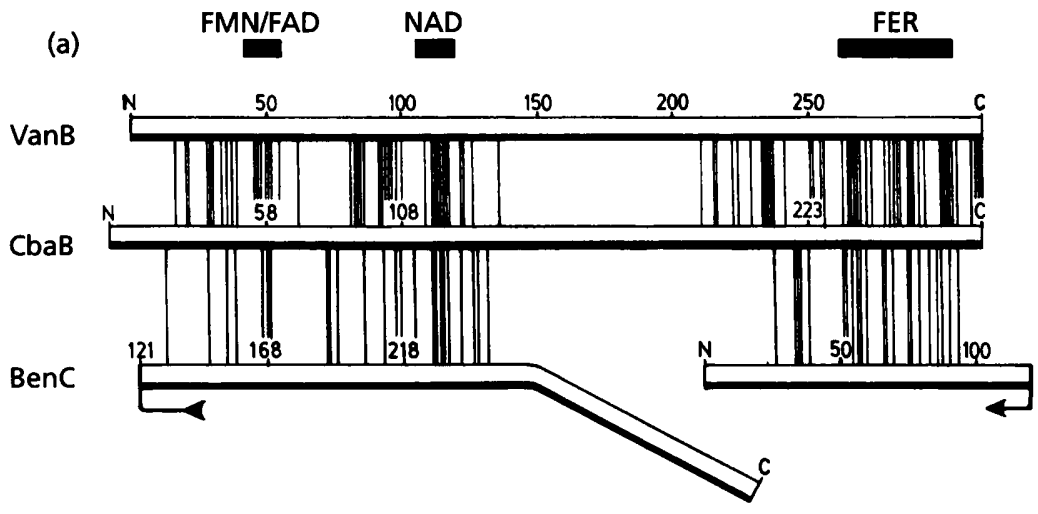

(b)
FMN/FAD-peptide

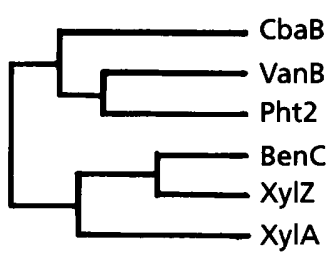

Class

\section{IA}

\section{IA}

IA

IB

IB

Mono
FER-Peptide

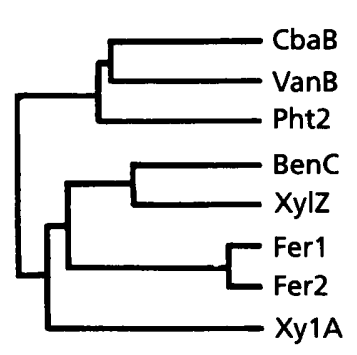

Class

IA

IA

IA

IB

IB

Plant

Plant
Fig. 6. (a) Scheme of the complete alignment of the FMN/FAD-isoalloxazine, NAD-ribose- and [2Fe-2S $]_{\mathrm{Fd}}$-binding domains of reductase components of class IA (VanB, $\mathrm{CbaB}$ ) and class IB (BenC) oxygenases. The amino acid sequences are represented by bars with amino acid numbers indicated. Vertical lines represent conserved amino acid pairs. The VanB and $\mathrm{CbaB}$ sequences begin with N-terminal FMN/FAD-isoalloxazine- and NAD-ribose-binding domains, followed by C-terminal ferredoxin $[2 \mathrm{Fe}-2 \mathrm{~S}]_{\mathrm{Fd}}{ }^{-}$ binding domains. The BenC sequence begins with an $\mathrm{N}$-terminal ferredoxin $[2 \mathrm{Fe}-2 \mathrm{~S}]_{\mathrm{Fd}}{ }^{-}$ binding domain and ends with a C-terminal FMN/FAD-isoalloxazine- and NAD-ribosebinding domain. (b) Cluster diagrams showing potential evolutionary relationships between amino acid sequences representing the two parts of the reductase proteins of oxygenases. The left diagram is based on pairwise similarity scores between the approximately 200 amino acids spanning the FMN/FAD-isoalloxazine- and NAD-ribosebinding domains. The right diagram is based on similarities between the approximately 110 amino acids spanning the ferredoxin $[2 \mathrm{Fe}-2 \mathrm{~S}]_{\mathrm{Fd}}$-binding domain. Mono signifies a monooxygenase class. stream of $c b a B$ encodes a $42 \mathrm{kDa}$ protein (Nakatsu \& Wyndham, 1993). These preliminary results suggest this region of Tn5271 encodes a 3-chlorobenzoate 3,4-dihydrodiol dehydrogenase. Sequencing, database comparisons, and expression studies are continuing on this ORF in an effort to identify the product and determine its similarity to known dehydrogenases.

\section{DISCUSSION}

Earlier expression studies (Nakatsu \& Wyndham, 1993), using constructs with various fragments of Tn 5271 placed under the control of either the hybrid tac promoter of the broad-host-range plasmid pMMB66HE or the T7 promoter of pGEM3Zf, showed that two protein products (51 and $42 \mathrm{kDa}$ ) were formed from clones capable of restoring 3-chlorobenzoate degradation. The pattern of metabolites formed by clones that did not express the $42 \mathrm{kDa}$ protein was consistent with its assignment as a dihydrodiol dehydrogenase. This protein was expressed from a cloned S $c a$ I-HindIII fragment extending from position $5 \cdot 7$ to $9.8 \mathrm{~kb}$ (Fig. 1), which includes the $c b a B$ gene and the ORF downstream of $c b a B$. However, based on the size of the expressed protein $(42 \mathrm{kDa})$ we assume that it was the product of the downstream ORF and not of $c b a B$. No evidence for expression of a $32 \mathrm{kDa}$ protein corresponding in size to $\mathrm{CbaB}$ was found, using two different expression vectors. However, the evidence presented here for the elimination of 3-chlorobenzoate metabolism in vivo in cells carrying the mutated $c b a B$ gene on pBRCN15 and pBRCN12 indicates that the $c b a B$ gene must be expressed for dioxygenase activity. Therefore, we contend that 3-chlorobenzoate 3,4-dioxygenase is a twocomponent enzyme made up of a terminal dioxygenase $(\mathrm{CbaA})$ plus a reductase $(\mathrm{CbaB})$ and that the $\mathrm{CbaB}$ reductase is unstable and is degraded during the extraction procedure used to investigate expression in vivo.

Characterization of the presumptive $\mathrm{CbaC}$ dehydrogenase encoded downstream of $c b a B$ remains to be completed. Expression of the entire operon in natural hosts of Tn5271 probably depends on the activity of an upstream promoter contained within an A $+\mathrm{T}$-rich region of $142 \mathrm{bp}$ $(60 \% \mathrm{~A}+\mathrm{T})$, located between the NotI site (Fig. 1) and the putative ribosome-binding site of $c b a A$ (complete sequence not shown). There are no obvious similarities between this sequence and the known promoter sequences of other multi-component oxygenases, including the $\mathrm{P}_{\mathrm{m}}$ or $\mathrm{P}_{\mathrm{u}}$ promoters of the TOL plasmid and the putative promoter regions of the pht12345 and van $A B$ operons. Characterization of the $c b a A B$ promoter is in progress.

The biochemical classification scheme for bacterial oxygenases described by Batie et al. (1991) is continually being reinforced as new aromatic ring oxygenases are characterized (Bünz \& Cook, 1993; Romanov \& Hausinger, 1994). As the amino acid sequences of these oxygenases become known, evidence is accumulating that the biochemical classification has a strong evolutionary basis (Neidle et al., 1991; Harayama et al., 1992). In this study the class IA oxygenases have been united in a single lineage on the basis of similarities between their component amino acid sequences. Relative evolutionary 


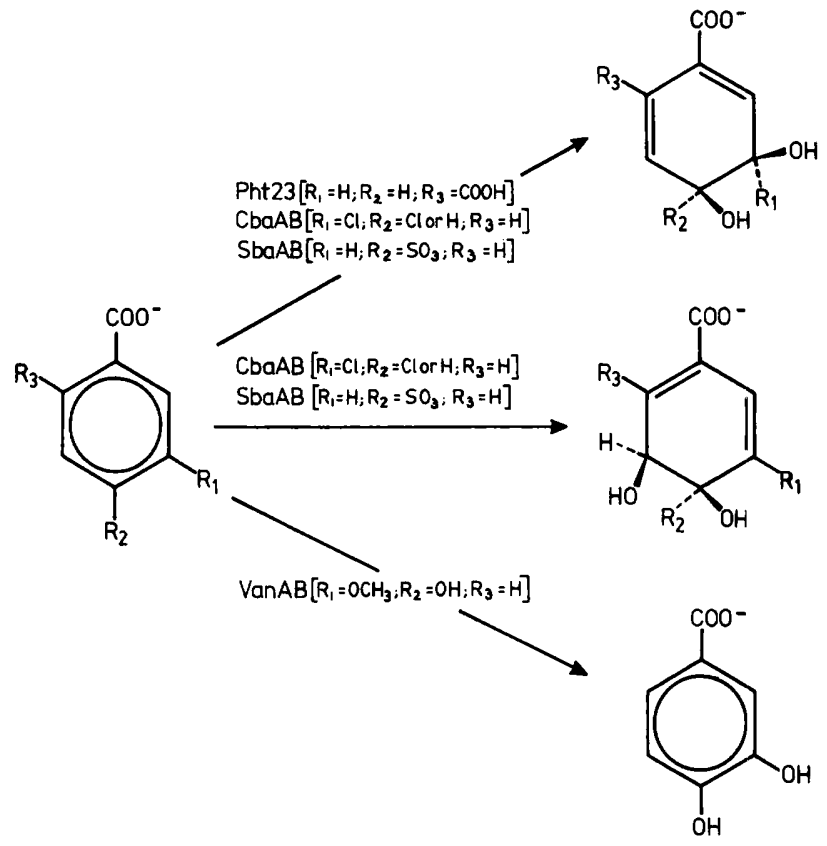

Fig. 7. Reactions carried out by the class IA oxygenases. The requirement for a carboxyl group on the ring is common to all the sequenced class IA oxygenases. Substrate R-groups vary as indicated for the phthalate dioxygenase (Pht23), chlorobenzoate dioxygenase (CbaAB), sulphobenzoate dioxygenase (SbaAB) and vanillate demethylase (VanAB) reactions. Chlorobenzoate dioxygenase ( $\mathrm{CbaAB}$ ) is active with 3-chloro- and 3,4-dichloro-benzoates (Nakatsu \& Wyndham, 1993). The dihydrodiol products of Pht23 and SbaAB are converted to 4,5-dihydroxyphthalate and protocatechuate, respectively (Nomura et al., 1992; Locher et al., 1991). The dihydrodiol products of $\mathrm{CbaAB}$ are converted to protocatechuate and 4-chloroprotocatechuate (Nakatsu \& Wyndham, 1993). VanAB is reported to catalyse a reaction mechanism involving introduction of one atom of oxygen at the methyl-carbon at position $\mathrm{R} 1$ to generate a hemiacetal intermediate that spontaneously decomposes to protocatechuate and formaldehyde (Brunel \& Davison, 1988).

distances determined from pairwise amino acid sequence comparisons correspond well with the biochemical classification system of Batie et al. (1991). The terminal oxygenase components of class IA, in the region containing the $[2 \mathrm{Fe}-2 \mathrm{~S}]_{R}$-binding domains, are more closely related to each other than to the terminal oxygenases of the other classes (Figs 3 and 4 ). The reductase components of the class IA oxygenases (Fig. 5) show similar cofactorand iron-binding domains to the class IB reductases and xylene monooxygenase reductase. However, the orientation of the chloroplast-type $[2 \mathrm{Fe}-2 \mathrm{~S}]_{\mathrm{Fd}}$-binding domain and the FMN/FAD-isoalloxazine- and NAD-ribosebinding domains are reversed in all of the known class IA reductases, including the 3-chlorobenzoate dioxygenase reductase described here, compared to other dioxygenase reductases (Fig. 6). This reversal has been attributed to alternative fusions of the three distinct domains of these reductases (Correll $e$ t al., 1992). Determination of the crystal structure of phthalate dioxygenase reductase has shown that alternative fusions of the cofactor- and iron- binding domains of the reductases can be achieved by short peptide linkers that cause minimal disturbance to the structure of the reductase (Correll et al., 1992). This would suggest that alternative fusions may arise frequently during the evolution of these electron transport systems. However, the sequence comparisons and relative evolutionary distance diagrams presented in Figs 4 and 6 indicate that the class IA oxygenases form a unified lineage that has conserved the unique fusion arrangement of the reductase domains despite considerable divergence in sequence and function. This suggests that the observed fusion of the cofactor- and iron-binding domains of the class IA dioxygenase reductases probably occurred early in the evolution of the electron transport systems of bacterial oxygenases.

There is good evidence that operons coding for pollutant biodegradation are assembled in a stepwise manner from existing catabolic genes (van der Meer et al., 1992). It is possible that reductase genes for one class of oxygenase may be recruited into another class. Recently a reductase gene $(p c p D)$ has been described that encodes an amino acid sequence that is $>55 \%$ similar to the VanB and Pht 2 class IA reductases (Lange et al., 1994). This gene is located 13 nucleotides downstream from the pentachlorophenol 4-monooxygenase gene $p c p B$ of Flavobacterium sp. strain ATCC 39723, suggesting it is co-transcribed. Unlike the class IA oxygenases, however, pentachlorophenol 4-monooxygenase ( $\mathrm{PcpB}$ ) is a NADPH-dependent flavoprotein monooxygenase (Orser et al., 1993). This observation suggests that the class IA reductases may have been recruited for a variety of aromatic ring oxidation reactions.

The unique features of the class IA dioxygenases may be used in the future to recruit new oxygenases to this class. For example, the 4-sulphobenzoate 3,4-dioxygenase of C. testosteroni $\mathrm{T} 2$ belongs to this class on the basis of biochemical studies (Locher et al., 1991). Based on the $\mathrm{N}$ terminal 35 amino acids sequenced from the terminal oxygenase subunit, compared to the corresponding sequences of the $\mathrm{CbaA}$ and $\mathrm{Pht} 3$ proteins, this dioxygenase belongs to the class IA lineage defined here. It remains for complete sequencing of this dioxygenase and the associated reductase to establish its evolutionary similarity. Isolation and sequencing of dehalogenating class IA dioxygenases other than $\mathrm{CbaAB}$, for example the 4chlorophenylacetate 3,4-dioxygenase of Pseudomonas sp. CBS3 (Markus et al., 1986; Schweizer et al., 1987) is also expected to strengthen the conclusions presented here.

Both monooxygenase (vanillate demethylase) and dioxygenase enzymes belong to the class IA oxygenases. The terminal oxygenase components of these enzymes are more closely related to one another than to dioxygenases from different classes, suggesting that biochemical variation may arise rapidly within a single lineage of oxygenase genes. Sequence comparisons have not shed light on the important amino acid domain(s) determining substrate binding and monooxygenase versus dioxygenase activity. Biochemical studies have shown that the aromatic hydrocarbon dioxygenases may catalyse 
monooxygenase reactions (Wackett $e t$ al., 1988), and the 4-methoxybenzoate monooxygenase of $P$. putida can dihydroxylate the alkyl side chain of 4-methoxystyrene (Wende et al., 1989). Therefore, the fate of the oxygen atoms at the active site of these enzymes may not be as important an evolutionary character as the orientation of attack on the substituted aromatic ring.

We speculated earlier that there may be an evolutionary relationship between the 4-sulphobenzoate 3,4-dioxygenase and the 3-chlorobenzoate 3,4-dioxygenase because both host organisms belong to the Comamonadaceae, and because the relative stereospecificity of the reactions was similar (Nakatsu \& Wyndham, 1993). An examination of Fig. 7, showing the orientations of oxygen insertion into the four different substrates of the class IA oxygenases, supports this conclusion. The over-riding evolutionary constraint acting on the divergence of the class IA oxygenases would appear to be the requirement for a carboxyl group para to the site of oxygen insertion. The amino acid(s) of the dioxygenase component that orient the substrate within the active site and that presumably recognize the carboxyl group are not known, however they should be conserved in all class IA dioxygenase sequences. Conserved amino acids that may serve this function in the class IA dioxygenases are the three arginines at positions 329, 377 and 378 (Fig. 3, CbaA numbering). Arginines also make up three of the nine conserved residues in an alignment of the $\beta$-subunits of the class IB, IIB and III oxygenases that are required for substrate binding (Neidle et al., 1991). Further sequencing, mutagenesis and expression studies are required to establish the nature of active sites and the mechanistic and evolutionary relationships between dioxygenase and monooxygenase reactions in these enzymes.

\section{ACKNOWLEDGEMENTS}

The authors thank Dr Rama Singh, Bess Wong, Barbara Holland and Maysoon Salih for their assistance. The work reported here was supported by a Research Grant to R.C.W. from the Natural Sciences and Engineering Research Council of Canada.

\section{REFERENCES}

Altschul, S. F., Gish, W., Miller, M., Myers, E. W. \& Lipman, D. J. (1990). Basic local alignment search tool. J Mol Biol 215, 403-410.

Batie, C. J., Ballou, D. P. \& Correll, C. J. (1991). Phthallate dioxygenase reductase and related flavin-iron-sulphur containing electron transferases. In: Chemistry and Biochemistry of Flavoenzymes, pp. 544-554. Edited by F. Müller, Boca Raton: CRC Press.

Brunel, F. \& Davison, J. (1988). Cloning and sequencing of Pseudomonas genes encoding vanillate demethylase. J Bacteriol 170, $4924-4930$.

Bunz, P. V. \& Cook, A. M. (1993). Dibenzofuran-4,4a-dioxygenase from Sphingomonas sp. strain RW1: angular dioxygenation by a three-component enzyme system. J Bacteriol 175, 6467-6475.

Chan, T.-M., Hermodson, M. A., Ulrich, E. L. \& Markley, J. L. (1983). Nuclear magnetic resonance studies of two-iron-twosulphur ferredoxins. 2. Determination of the sequence of Anabaena variabilis ferredoxin II, assignment of aromatic resonances in proton spectra, and effects of chemical modifications. Biochemistry 22 , 5988-5995.

Correll, C. C., Batie, C. J., Ballou, D. P. \& Ludwig, M. L. (1992). Phthallate dioxygenase reductase: a modular structure for electron transfer from pyridine nucleotides to [2Fe-2S]. Science 258, 16041610.

Devereux, J., Haeberli, P. \& Smithies, O. (1984). A comprehensive set of sequence analysis programs for the VAX. Nucleic Acids Res 12, 387-395.

Erickson, B. D. \& Mondello, F. J. (1992). Nucleotide sequencing and transcriptional mapping of the genes encoding biphenyl dioxygenase, a multicomponent polychlorinated-biphenyl-degrading enzyme in Pseudomonas strain LB400. J Bacteriol 174, 2903-2912.

Fetzner, S., Muller, R. \& Lingens, F. (1992). Purification and some properties of 2-halobenzoate 1,2-dioxygenase, a two component enzyme system from Pseudomonas cepacia 2CBS. J Bacteriol 174, 279-290.

Frantz, B. \& Chakrabarty, A. M. (1986). Degradative plasmids in Pseudomonas. In: The Bacteria, vol. 10, pp. 295-323. Edited by J. R. Sokatch. New York: Academic Press.

Frantz, B. \& Chakrabarty, A. M. (1987). Organization and nucleotide sequence determination of a gene cluster involved in 3chlorocatechol degradation. Proc Natl Acad Sci USA 84, 44604464.

Furukawa, K., Hayase, N., Kazunari, T. \& Tomizuka, N. (1989). Molecular relationship of chromosomal genes encoding biphenyl/ polychlorinated biphenyl catabolism: some soil bacteria possess a highly conserved $b p b$ operon. J Bacteriol 171, 5467-5472.

Gibson, D. T. \& Subramanian, V. (1984). Microbial degradation of aromatic hydrocarbons. In: Microbial Degradation of Organic Compounds, pp. 181-252. Edited by D. T. Gibson. New York: Marcel Dekker.

Harayama, S., Rekik, M., Bairoch, A., Neidle, E. L. \& Ornston, L. N. (1991). Potential DNA slippage structures acquired during evolutionary divergence of Acinetobacter calcoaceticus chromosomal ben $A B C$ and Pseudomonas putida TOL pWW0 plasmid $x y l X Y Z$, genes encoding benzoate dioxygenases. J Bacteriol 173, 7540-7548.

Harayama, S., Kok, M. \& Neidle, E. L. (1992). Functional and evolutionary relationships among diverse oxygenases. Annu Rev Microbiol 46, 565-601.

Hickey, W. J. \& Focht, D. D. (1990). Degradation of mono-, di- and trihalogenated benzoic acids by Pseudomonas aeruginosa JB2. Appl Environ Microbiol 56, 3842-3850.

Irie, S., Doi, S., Yorifugi, T., Takagi, M. \& Yano, K. (1987). Nucleotide sequencing and characterization of the genes encoding benzene oxidation enzymes of Pseudomonas putida. J Bacteriol 169, 5174-5179.

Johnston, H. W., Briggs, G. G. \& Alexander, M. (1972). Metabolism of 3-chlorobenzoic acid by a pseudomonad. Soil Biol Biochem 4, 187-190.

Kurkela, S., Lehvaeslaiho, H., Palva, E. T. \& Teeri, T. H. (1988). Cloning, nucleotide sequence and characterization of genes encoding naphthalene dioxygenase of Pseudomonas putida strain NCIB9816. Gene 73, 355-362.

Lange, C. C., Edwards, A. R. \& Orser, C. S. (1994). Identification of two new genetic loci, $p c p D$ and $p c p \mathrm{R}$, involved in pentachlorophenol degradation by Flavobacterium sp. strain ATCC 39723. Abstracts of the American Society for Microbiology Annual Meeting, Las Vegas, NV, USA, Q-414, p. 461.

Locher, H. H., Leisinger, T. \& Cook, A. M. (1991). 4-Sulphobenzoate-3,4-dioxygenase. Purification and properties of a desul- 
phonative two-component enzyme system from Comamonas testosteroni T-2. Biochem J 274, 833-842.

Markus, A., Krekel, D. \& Lingens, F. (1986). Purification and some properties of component A of the 4-chlorophenylacetate-3,4dioxygenase from Pseudomonas species strain CBS. J Biol Chem 261, 12883-12888.

Mason, J. R. \& Cammack, R. (1992). The electron-transport proteins of hydroxylating bacterial dioxygenases. Annu Rev Microbiol 46, 277-305.

van der Meer, J. R., Eggen, R. I. L., Zehnder, A. J. B. \& de Vos, W. M. (1991). Sequence analysis of the Pseudomonas sp. strain P51 tcb gene cluster, which encodes metabolism of chlorinated catechols: evidence for specialization of catechol-1,2-dioxygenases for chlorinated substrates. J Bacteriol 173, 2425-2434.

van der Meer, J. R., de Vos, W. M., Harayama, S. \& Zehnder, A. J. B. (1992). Molecular mechanisms of genetic adaptation to xenobiotic compounds. Microbiol Rev 56, 677-694.

Morrice, N., Geary, P., Cammack, R., Harris, A., Beg, F. \& Aitken, A. (1988). Primary structure of protein B from Pseudomonas putida, member of a new class of $2 \mathrm{Fe}-2 \mathrm{~S}$ ferredoxins. FEBS Lett 231, 336-340.

Nakatsu, C. H. \& Wyndham, R. C. (1993). Cloning and expression of the transposable chlorobenzoate-3,4-dioxygenase genes of Alcaligenes sp. BR60. Appl Environ Microbiol 59, 3625-3633.

Nakatsu, C., Ng, J., Singh, R., Straus, N. \& Wyndham, C. (1991). Chlorobenzoate catabolic transposon $\operatorname{Tn} 5271$ is a composite class I element with flanking class II insertion sequences. Proc Natl Acad Sci US A 88, 8312-8316.

Neidle, E. L., Hartnett, C., Bonitz, S. \& Ornston, L. N. (1988). DNA sequence of the Acinetobacter calcoaceticus catechol 1,2-dioxygenase I structural gene cat $A$ : evidence for evolutionary divergence of intradiol dioxygenases by acquisition of DNA sequence repetitions. J Bacteriol 170, 4874-4880.

Neidle, E. L., Hartnett, C., Ornston, L. N., Bairoch, A., Rekik, M. \& Harayama, S. (1991). Nucleotide sequences of the Acinetobacter calcoaceticus ben $A B C$ genes for benzoate 1,2-dioxygenase reveal evolutionary relationships among multicomponent oxygenases. J Bacteriol 173, 5385-5395.

Nomura, Y., Nakagawa, M., Ogawa, N., Harashima, S. \& Oshima, Y. (1992). Genes in $P H^{\prime} T$ plasmid encoding the initial degradation pathway of phthalate in Pseudomonas putida. J Ferment Bioeng 74, 333-344.

Orser, C. S., Lange, C. C., Xun, L., Zahrt, T. C. \& Schneider, B. J. (1993). Cloning, sequence analysis, and expression of the Flavobacterium pentachlorophenol-4-monooxygenase gene in Escherichia coli. J Bacteriol 175, 411-416.

Otaka, E. \& Ooi, T. (1989). Examination of protein sequence homologies. V. New perspectives on evolution between bacterial and chloroplast-type ferredoxins inferred from sequence evidence. $J$ Mol Evol 29, 246-254.

Reineke, W. \& Knackmuss, H.-J. (1988). Microbial degradation of haloaromatics. Annu Rev Microbiol 42, 263-287.

Romanov, V. \& Hausinger, R. P. (1994). Pseudomonas aeruginosa 142 uses a three-component ortho-halobenzoate-1,2-dioxygenase for metabolism of 2,4-dichloro- and 2-chlorobenzoate. J Bacteriol 176, 3368-3374.
Sambrook, J., Fritsch, E. F. \& Maniatis, T. (1989). Molecular Cloning: a Laboratory Manual. Cold Spring Harbor, NY: Cold Spring Harbor Laboratory.

Savard, P., Peloquin, L. \& Sylvestre, M. (1986). Cloning of Pseudomonas sp. strain CBS3 genes specifying dehalogenation of 4-chlorobenzoate. $J$ Bacteriol 168, 81-85.

Sayler, G. S., Hooper, S. W., Layton, A. C. \& Henry King, J. M. (1990). Catabolic plasmids of environmental and ecological significance. Microb Ecol 19, 1-20.

Scholten, J. D., Chang, K. H., Babbitt, P. C., Charest, H., Sylvestre, M. \& Dunaway-Mariano, D. (1991). Novel enzymic hydrolytic dehalogenation of a chlorinated aromatic. Science 253, 182-185.

Schweizer, D., Markus, A., Seez, M., Ruf, H. H. \& Lingens, F. (1987). Purification and some properties of component $B$ of the 4-chlorophenylacetate-3,4-dioxygenase from Pseudomonas species strain CBS3. J Biol Chem 262, 9340-9346.

Suzuki, M., Hayakawa, T., Shaw, J. P., Rekik, M. \& Harayama, S. (1991). Primary structure of xylene monooxygenase: similarities to and differences from the alkane hydroxylation system. $J$ Bacteriol 173, 1690-1695.

Wackett, L. P., Kwart, L. D. \& Gibson, D. T. (1988). Benzylic monooxygenation catalyzed by toluene dioxygenase from Pseudomonas putida. Biochemistry 27, 1360-1367.

Wende, P., Bernhardt, F.-H. \& Pfleger, K. (1989). Substratemodulated reactions of putidamonooxin: the nature of the active oxygen species formed, and its reaction mechanism. Eur J Biochem 181, 189-197.

West, S. E. H. \& Iglewski, B. H. (1988). Codon usage in Pseudomonas aeruginosa. Nucleic Acids Res 16, 9323-9335.

Willems, A., DeLey, J., Gillis, M. \& Kersters, K. (1991). Comamonadaceae, a new family encompassing the Acidovorans rRNA complex, including Variovorax paradoxus gen. nov., comb. nov., for Alcaligenes paradoxus (Davis 1969). Int J Syst Bacteriol 41, 445-450.

Wyndham, R. C. \& Straus, N. A. (1988). Chlorobenzoate catabolism and interaction between Alcaligenes and Pseudomonas species from Bloody Run Creek. Arch Microbiol 150, 230-236.

Wyndham, R. C., Singh, R. K. \& Straus, N. A. (1988). Catabolic instability, plasmid gene deletion and recombination in Alcaligenes sp. BR60. Arch Microbiol 150, 237-243.

Wyndham, R. C., Cashore, A., Nakatsu, C. \& Peel, M. (1994). Catabolic transposons. Biodegradation 5, 323-342.

Yamaguchi, M. \& Fujisawa, H. (1982). Subunit structure of oxygenase component in benzoate-1,2-dioxygenase system from Pseudomonas arvilla C-1. J Biol Chem 257, 12497-12502.

Yanisch-Perron, C., Vieira, J. \& Messing, J. (1985). Improved M13 phage cloning vectors and host strains : nucleotide sequences of the M13mp18 and pU19 vectors. Gene 33, 103-119.

Zylstra, G. J. \& Gibson, D. T. (1989). Toluene degradation by Pseudomonas putida $\mathrm{F} 1$ : nucleotide sequence of the todC1C2B ADE genes and their expression in E. coli. J Biol Chem 264, 14940-14946.

Received 5 July 1994; revised 19 September 1994; accepted 13 October 1994. 